\title{
Comportamento Precorrente Auxiliar: Efeitos do Número de Dimensões Discriminativas da Tarefa ${ }^{1}$
}

\author{
Jorge M. Oliveira-Castro \\ Ana Paula Martins de Campos \\ Universidade de Brasília
}

\begin{abstract}
RESUMO - Trabalhos anteriores mostraram que o nível de complexidade de tarefas de pares associados pode ser medido com base na quantificação das probabilidades programadas de reforço na presença das duas diferentes dimensões discriminativas das tarefas. No Experimento 1 (seis estudantes) do presente trabalho, foram investigados possíveis efeitos diferenciais de manipulações nas probabilidades de reforço para essas duas dimensões (aprender $n$ respostas associadas ao mesmo evento versus aprender $n$ respostas associadas cada uma a um evento diferente). No Experimento 2 (16 estudantes), o número de dimensões discriminativas do primeiro membro dos pares variou de dois (forma e posição) a quatro (forma, posição, cor, e forma secundária). De forma geral, os resultados sugerem que o desempenho melhora com aumentos no nível de correspondência unívoca entre estímulos discriminativos e respostas. Os efeitos observados podem ser explicados com base nas mudanças nas probabilidades programadas de reforço na presença das diferentes dimensões.
\end{abstract}

Palavras-chave: análise do comportamento; comportamento precorrente; complexidade de tarefas; dimensões de estímulo; pares-associados.

\section{Auxiliary Precurrent Behavior: Effects of the Number of Discriminative Dimensions in the Task}

\begin{abstract}
Previous studies have shown that the level of complexity of paired-associates tasks can be measured on the basis of the quantification of programmed reinforcement probabilities in the presence of the two discriminative dimensions of these tasks. In Experiment 1 (six students) of the present paper, possible differential effects of changes in reinforcement probability for these two dimensions were investigated (to learn $n$ responses associated to the same events versus to learn $n$ responses each one associated to a different event). In Experiment 2 (16 students), the number of discriminative dimensions of the first member of the associated pairs varied from two (shape and position) to four (shape, position, color, and secondary shape). In general, results suggest that performance increases with increases in the level of univocal correspondence between discriminative stimuli and responses. The observed effects can be explained on the basis of changes in the programmed probabilities of reinforcement in the presence of different dimensions.
\end{abstract}

Key words: behavior analysis; precurrent behavior; task complexity; stimuli dimensions; paired-associates.

Quando alguém é descrito como resolvendo problemas aritméticos mentalmente ou "na cabeça", parte do que se está afirmando é que a pessoa resolve os problemas sem escrever ou olhar os números, relacionados a passos intermediários do cálculo, no papel ou quadro-negro e sem falar ou ouvir tais números. Da mesma forma, quando alguém é descrito como tendo memorizado um número de telefone, parte do que se está afirmando é que a pessoa é capaz de discar o número sem ter que consultar a agenda ou lista telefônica ou perguntar a alguém. Em ambos os casos, algumas respostas que ocorriam e eram necessárias para um bom desempenho, tais como consultar a agenda ou escrever passos intermediários de cálculos aritméticos, param de ocorrer. Desempenho

1 Os autores agradecem ao CNPq pelo apoio (bolsas de pesquisador e iniciação científica) e a Moema B. Dias e Thiago L. Carneiro pelo auxílio na coleta de dados. Partes dessa pesquisa foram apresentadas na 52a. Reunião Anual da Sociedade Brasileira para o Progresso da Ciência, Brasília, 2000.

2 Endereço: Universidade de Brasília, Instituto de Psicologia, Campus Universitário Darcy Ribeiro, Brasília, DF, Brasil 70910-900. E-mail: jocastro@unb.br correto ocorre, depois de algum treino, sem a emissão destas respostas, as quais são eliminadas da seqüência original de respostas. Exemplos desse tipo de comportamento, denominado "precorrente auxiliar" (ver explicação adiante), podem ser identificados em quase qualquer tarefa, tais como olhar para os pedais quando aprendendo a dirigir, ou olhar para o teclado quando aprendendo a datilografar, ou ouvir o instrutor quando aprendendo a pronunciar uma nova palavra estrangeira, ou consultar uma tabuada quando aprendendo a multiplicar.

Essa análise, a qual indica que alguns comportamentos deixam de ocorrer com o aumento do treino, tem várias implicações para interpretações de "eventos privados", discutidas em trabalhos anteriores. Em linhas gerais, sugeriuse que uma interpretação não denotacionista de conceitos psicológicos pode ser menos confusa e mais útil do que a prática de interpretar termos psicológicos como se referindo a eventos privados. Tal proposta encorajaria a investigação das condições que influenciam a diminuição desse tipo de comportamento e a formação de repertórios de nível superior (Oliveira-Castro, 1992; 1993; 2000b). 
Apesar da abrangência desse tipo de comportamento precorrente auxiliar, que ocorre em quase qualquer tarefa, poucas pesquisas têm investigado o fenômeno de forma sistemática. O trabalho de Blessing e Anderson (1996) pode ser mencionado como uma exceção, no qual os autores procuraram investigar como as pessoas aprendem a pular passos em uma tarefa. Para isso, os autores utilizaram uma tarefa de "matemática artificial", na qual os participantes deviam procurar solucionar problemas que simulavam equações algébricas com símbolos e formas pouco familiares. Durante os experimentos os participantes podiam consultar as regras de utilização dos símbolos e deviam redigir a solução dos problemas. Os autores observaram decréscimos, negativamente acelerados, no número de respostas de consulta às regras (com eventual desaparecimento das mesmas) e na quantidade de linhas escritas para resolver os problemas. Blessing e Anderson (1996) explicaram os resultados obtidos com base na teoria racional de controle adaptativo de pensamento (ACT-R; Anderson, 1993), de acordo com a qual conhecimento procedural novo deriva de conhecimento declarativo na forma de exemplos, com a formação de regras de nível superior.

Adotando um referencial teórico diferente, esse tipo de comportamento, que pode deixar de ocorrer depois de algum treino, pode ser interpretado, mais tecnicamente, como um tipo de comportamento precorrente (Oliveira-Castro, 2000b; Oliveira-Castro, Coelho \& Oliveira-Castro, 1999), um conceito adotado por Skinner $(1953 ; 1957 ; 1968 ; 1969)$ para se referir a respostas que aumentam a probabilidade de outras respostas (correntes) ocorrerem ou serem reforçadas. Essas respostas, tais como consultar um número de telefone na agenda, podem aumentar, pelo menos no início do treino, a probabilidade de outras respostas serem reforçadas, tais como discar o número correto. Esse tipo de comportamento precorrente, que tem sido nomeado de "auxiliar" (Carvalho, 2000; Coelho, 1996, 2000; Ferreira, 2001; Flores, 1997, 2003; Gosch, 2000; Souza, 1997), é não requerido pelas contingências programadas, sinalizado, e ocorre em situações que possibilitam a transferência de função de estímulo (Oliveira-Castro \& cols., 1999).

Oliveira-Castro e cols. (1999) desenvolveram um procedimento de pares-associados, análogo ao exemplo do número de telefone mencionado acima, para investigar as variáveis que influenciam a diminuição da duração das respostas precorrentes auxiliares. Em uma variação deste procedimento, ao se apresentar uma forma arbitrária como primeiro membro do par associado, os participantes podiam consultar o segundo membro do par, geralmente composto por uma sequiência de caracteres arbitrários, antes de digitá-lo. A tarefa dos participantes era memorizar o segundo membro de cada par, isto é, eles deveriam ser capazes de digitar os caracteres que formavam o segundo membro de cada par, na presença do primeiro membro de cada par, sem consultar uma tela auxiliar. Cada sessão experimental envolvia geralmente quatro a oito pares, os quais eram apresentados em blocos de tentativas contendo uma apresentação de cada par. Como os pares se repetiam no decorrer da sessão, os participantes podiam aprender a digitar os segundos membros dos pares, ao serem apresentados os primeiros membros, sem consultar o auxílio. A duração da resposta precorrente auxiliar, ou seja, o tempo que os participantes gastaram consultando a tela de auxílio, foi medida para cada par em cada tentativa. Resultados de 19 experimentos (Carvalho, 2000; Coelho, 1996, 2000; Ferreira, 2001; Flores, 1997; Gosch, 2000; Oliveira-Castro, 1993; Oliveira-Castro, Faria, Dias \& Coelho, 2002; Oliveira-Castro \& cols., 1999; Souza, 1997), usando diferentes números de pares e diferentes tarefas, indicaram que a duração da resposta precorrente auxiliar para cada resposta corrente correta (digitar o segundo membro do par) decresceu como uma função inversa do logaritmo dos blocos de tentativas. Em outras palavras, com o aumento do número de blocos de tentativas, a duração de consulta necessária para digitar corretamente os segundos membros dos pares diminuía como uma função negativamente acelerada. Tais resultados foram descritos de acordo com a seguinte equação:

Duração/Correta $=b-a(\log$ Tentativas) (1)

onde $b$ e $a$ são parâmetros obtidos empiricamente que podem ser interpretados, respectivamente, como a duração estimada de resposta precorrente auxiliar por correta no primeiro bloco de tentativas e a taxa de decréscimo entre blocos de tentativas da duração da resposta precorrente auxiliar por correta. A tentativa estimada para memorização de todos os pares, isto é, o bloco de tentativas no qual a duração da resposta precorrente auxiliar seria igual a zero (sem erros), pode ser obtido por $b / a$. A área da função, obtida por $b^{2} / 2 a$, pode ser usada como medida global de desempenho, pois pode ser interpretada como a duração total estimada de resposta precorrente auxiliar necessária para respostas correntes corretas sem respostas auxiliares, ou seja, uma estimativa da duração total de respostas auxiliares necessárias para o aprendizado de cada resposta corrente. Esta tem sido a medida mais comumente empregada nas pesquisas experimentais sobre comportamento precorrente auxiliar.

Oliveira-Castro e cols. (1999) relataram aumentos no valor da área da função, calculada com base na Equação 1, com aumentos no número de respostas diferentes em cada par (número de caracteres diferentes que formavam cada conjunto de cinco dígitos), em cada posição (número de caracteres diferentes ocorrendo em cada uma de cinco posições entre pares) e no conjunto total a ser memorizado (número de caracteres diferentes que formavam todos os pares e posições). Com base nesses resultados, os autores propuseram que o nível de complexidade de uma tarefa seja medido com base na quantificação das contingências programadas de reforço na tarefa. No caso da tarefa de pares associados adotada, as contingências especificavam que uma determinada resposta (digitar um caractere) seria correta somente se ela ocorresse na presença da forma correta e na posição correta (i.e., primeira, segunda, etc.). Forma e posição, portanto, eram duas dimensões funcionais na tarefa, pois a consequiência apresentada após cada resposta (“Certo!" ou "Errado!") poderia mudar dependendo delas. As manipulações realizadas no experimento, ou seja, número de respostas diferentes por par, posição e no conjunto total, poderiam ser quantificadas da seguinte maneira: (a) Frequiência média programada de reforço (FMR), obtida com base na divisão do número de respostas corretas possíveis em qualquer bloco de tentativas pelo número de respostas diferentes; (b) Probabilidade média programada de reforço para qualquer resposta dada uma forma (PRForma), obtida pela divisão da frequiência média de reforço 
dada uma forma pelo número de respostas corretas possíveis dada uma forma; e (c) Probabilidade média programada de reforço para qualquer resposta dada uma posição (PRPos), obtida pela divisão da frequiência média de reforço dada uma posição pelo número de respostas corretas possíveis dada uma posição (Oliveira-Castro \& cols., 1999; Oliveira-Castro \& cols., 2002). A expressão complexidade discriminativa tem sido utilizada para distinguir mudanças nestas variáveis, as quais estão relacionadas às ocasiões adequadas para emitir certos movimentos (de certa forma, triviais para participantes adultos), de mudanças, por exemplo, no tipo e complexidade dos movimentos exigidos na tarefa (complexidade topográfica) (Oliveira-Castro \& cols., 1999).

Variações sistemáticas nos valores de PRForma, PRPos e FMR, baseadas em alterações separadas dos numeradores e denominadores destas medidas, foram realizadas em diferentes experimentos, produzindo os efeitos preditos, isto é, aumentos nos valores destas variáveis estiveram associados a diminuições nos valores de área (Oliveira-Castro \& cols., 1998b; Oliveira-Castro \& cols., 1997; Oliveira-Castro \& cols., 1996a; Oliveira-Castro \& cols., 1996b). Além disto, aumentos na frequiência programada de reforço para seqüências de respostas, mantendo PRForma, PRPos e FMR constantes (usando números para representar as formas e letras para representar os caracteres, os pares eram 1-ABCDE, 2-BCDEA, 3-CDEAB, 4-DEABC comparados com 1-ABCDE, 2-BDAEC, 3-CEDBA, 4-ECBAD), produziram diminuições nos valores de área (Oliveira-Castro \& cols., 1998a). OliveiraCastro \& cols. (2002) descreveram os resultados desses seis experimentos, os quais indicaram, em conjunto, efeitos sistemáticos da complexidade da tarefa aferida com base na quantificação das contingências programadas de reforço. Além disso, uma meta-análise dos dados desses experimentos indicou que PRForma apresentou maiores efeitos sobre o desempenho do que PRPos e FMR.

Em todos os experimentos realizados até o momento, as tarefas incluíam duas dimensões discriminativas, pois respostas eram corretas quando ocorriam na presença da forma (primeiro membro do par associado) correta e na posição (i.e., primeira, segunda, etc.) correta dentro da seqüência de caracteres que compunham o segundo membro do par associado. Usando números para representar as formas e letras para representar os caracteres, no caso de pares como "1-ABCDE" e "2-YWXZ", a resposta "W", por exemplo, só seria considerada correta se ocorresse na presença da forma "2" e na segunda posição dentro da seqüência "YWXZ". A maioria das tarefas do nosso dia a dia parece envolver mais de duas dimensões, como seria o caso de discar o telefone para falar com alguém que conhecemos e convivemos muito. Nesse caso, a resposta de discar não dependeria apenas do nome (análogo à forma nos exemplos) e dos números nas posições corretas (análogo aos caracteres nos exemplos), mas também de outros fatores, tais como, a hora do dia. Dependendo do horário, a relação nome e número de telefone pode ser diferente, isto é, o número a ser discado com relação ao mesmo nome pode depender da hora do dia, estando a pessoa tipicamente no trabalho ou na residência. Nesse caso, teríamos um exemplo de uma terceira dimensão discriminativa, o horário, que determinaria quais respostas de discar estariam corretas ou incorretas.
Os experimentos aqui relatados investigaram os efeitos do número de dimensões discriminativas na tarefa sobre o processo de aprendizagem, medido com base na diminuição da duração do comportamento precorrente auxiliar. Nos Experimentos 1 e 2 foram comparadas tarefas com uma, duas e três dimensões discriminativas.

\section{Experimento 1}

Antes de se investigar os possíveis efeitos do número de dimensões discriminativas da tarefa parece ser necessário saber se manipulações no número de diferentes dimensões têm efeitos semelhantes sobre o comportamento. Por exemplo, será que mudanças no número de formas têm o mesmo efeito que mudanças no número de posições? Será que aprender uma tarefa composta por um único par com oito caracteres é equivalente a aprender uma tarefa composta por oito pares cada um com um único caractere? Em ambos os casos, em princípio, a pessoa teria que aprender oito respostas. O presente experimento foi planejado para responder a tais perguntas. Para isso, em diferentes condições experimentais os participantes foram submetidos a tarefas de pares associados compostas por: 1) uma forma e oito posições; 2) oito formas e uma posição; 3) duas formas e quatro posições; 4) quatro formas e duas posições; e 5) quatro formas, duas posições e duas cores.

\section{Método}

\section{Participantes}

Participaram do experimento seis alunos da Universidade de Brasília (três mulheres), com idade média de 19,8 anos $(D P=1,6)$. Receberam $0,5 \%$ da menção final para cada hora de participação em disciplinas do Departamento de Processos Psicológicos Básicos do Instituto de Psicologia, podendo obter um máximo de 5\% (i.e., 10 horas) da menção final por disciplina.

\section{Material}

O experimento foi realizado em um microcomputador (486 DX2 - 80 MHZ) com tela colorida e mouse, localizado em uma cabine $(2 \times 2 \mathrm{~m})$ com isolamento acústico. $\mathrm{O}$ programa de execução do experimento foi elaborado em linguagem Delphi 2.0. Todos os conjuntos foram criados utilizando o programa Paint Brush para Windows 95. A Figura 1 apresenta as formas e conjuntos de caracteres usados para compor os pares associados em cada uma das condições experimentais.

\section{Procedimento}

No início de cada sessão eram apresentadas ao participante, na tela do computador, as seguintes instruções:

Agradecemos a sua participação neste experimento sobre memória. A sua tarefa consiste em memorizar alguns códigos, cada um associado a um conjunto. Leia com atenção as instruções a seguir. Um conjunto aparecerá numa tela. Clique com 


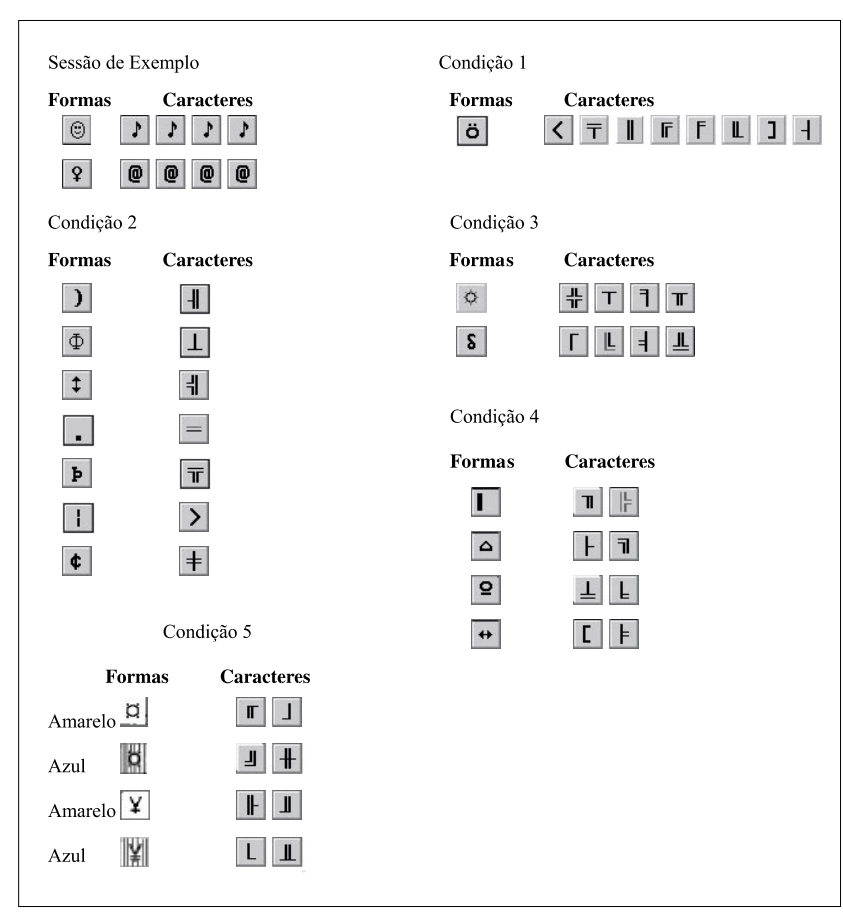

Figura 1. Pares de formas e caracteres utilizados em cada uma das condições do Experimento 1. Todas as formas e caracteres eram desenhadas em preto sobre fundo cinza, com exceção da Condição 5, na qual a cor do fundo das formas era amarela ou azul.

o mouse em "AUXÍLIO" para ver o código correspondente ao conjunto. Quando estiver pronto para escrever o código clique em "SAIR". Para escrever, clique em um componente do código de cada vez. Atenção! Você não poderá corrigir suas respostas. Se todos os componentes do código estiverem corretos, aparecerá a palavra "CORRETO!" na parte inferior da tela. Se qualquer um dos componentes estiver errado aparecerá a palavra “ERRADO!'. Os conjuntos se repetirão no decorrer da sessão e estarão sempre associados aos mesmos códigos. A sua tarefa, portanto, é memorizar o código correspondente a cada conjunto. Você poderá utilizaro "AUXÍLIO” sempre que julgar necessário. Porém, cada vez que você escrever, com o mouse, um código corretamente sem o "AUXÍLIO”, você ganhará um ponto no marcador à esquerda da tela. Após a apresentação da palavra "CORRETO!" ou "ERRADO!', um novo conjunto será apresentado e você deverá seguir os mesmos passos descritos acima. Clique em "OK” para começar.

Após o participante ter lido as instruções, pressionava com o mouse em "OK” (ícone localizado abaixo das instruções) e iniciava a sessão. A sequiência de apresentação de um par associado forma-caracteres era a seguinte: na parte superior da tela principal aparecia uma forma e na parte inferior um painel (semelhante a um teclado) com 16 a 20 caracteres, dentre os quais estavam os caracteres a serem utilizados como "resposta" na sessão (segundo membro do par). Para saber os caracteres correspondentes à forma apresentada, o participante deveria consultar a tela de auxílio pressionando com o mouse em "AUXÍLIO". Após um intervalo de 0,5 s, aparecia a tela de auxílio com os caracteres. O tempo de acesso a esta tela era livre. O participante pressionava com o mouse em "SAIR" e, após $0,5 \mathrm{~s}$, voltava à tela principal, já sem a forma em sua parte superior, onde os caracteres deveriam ser digitados pressionando com o mouse as teclas do painel localizado na parte inferior da tela ("teclado"). Após $0,5 \mathrm{~s}$, se os caracteres digitados estivessem corretos, aparecia a palavra "CERTO!" e um som agudo era apresentado. Se qualquer dos caracteres estivesse errado, aparecia a palavra "ERRADO!" e um som grave era apresentado. Se os caracteres fossem digitados corretamente sem consulta à tela de auxílio, um ponto era acrescido a um marcador no canto inferior esquerdo da tela. Os pontos obtidos não tinham qualquer relação programada com outros reforçadores ou conseqüências. Depois de 0,5 s, uma nova forma era apresentada, começando uma nova tentativa. A cada nova tentativa, as teclas do painel (i.e., "teclado") apareciam, aleatoriamente, em uma posição diferente do mesmo para evitar qualquer influência da posição das teclas (a discriminação deveria basear-se nos caracteres e não nas suas posições no "teclado"). Cada seqüência de caracteres (i.e., segundo membro dos pares) sempre estava associada à mesma forma (i.e., primeiro membro). As sessões eram compostas por blocos de tentativas, cada um contendo uma apresentação em sequiência aleatória de todos os pares sem repetição.

Sessão de exemplo: Nesta sessão o experimentador permanecia na sala esclarecendo dúvidas quanto às instruções e procedimento. Eram apresentados apenas dois pares associados que continham cada um: uma forma e um caractere repetido quatro vezes, diferentes dos que foram usados posteriormente. Ao término da sessão, o participante retirava-se da sala para que o experimentador preparasse a sessão seguinte.

Sessões experimentais: Durante essas sessões o experimentador permanecia fora da cabine de coleta de dados. $\mathrm{O}$ experimentador só retornava à cabine para preparar a sessão seguinte ou para solucionar eventuais problemas com o computador. Todas as sessões experimentais terminavam quando o participante atingia qualquer um dos seguintes critérios: 1) digitar, por duas vezes consecutivas, todos os pares da sessão corretamente sem consultar o auxílio (i.e., dois blocos de tentativas sem consulta e sem erro); ou 2) transcorridos 24 blocos de tentativas.

As duas primeiras sessões experimentais, Condição 1 e Condição 2, ocorreram logo após o término da sessão de exemplo (no mesmo dia). A Condição 1 (uma dimensão: posição) foi formada por uma forma (um par associado) e oito posições (i.e., oito caracteres diferentes). A Condição 2 (uma dimensão: forma) foi formada por oito formas (oito pares associados) e uma posição (i.e., um caractere diferente associado a cada forma) (ver Figura 1).

As três últimas sessões experimentais eram realizadas em um mesmo dia, um a quatro dias após as primeiras sessões. A Condição 3 (duas dimensões: forma e posição) foi composta por duas formas (dois pares associados) e quatro posições (i.e., quatro caracteres diferentes associados a cada forma). A Condição 4 (duas dimensões: forma e posição) foi composta por quatro formas (quatro pares) e duas posições (i.e., dois caracteres diferentes associados a cada forma). A Condição 5 (três dimensões: forma, posição e cor) foi composta por duas formas que apareciam em duas cores (quatro pares), e duas posições (i.e., dois caracteres diferentes associados a cada forma de cada cor) (ver Figura 1). 
A ordem de apresentação das Condições 1 e 2 e das Condições 3 e 4 foi invertida para três dos seis participantes. Duas versões de cada condição experimental foram criadas, usando conjuntos de formas e caracteres diferentes, sendo que três dos participantes foram expostos a uma versão enquanto os outros três passaram pela outra versão. Essas versões foram criadas com o objetivo de minimizar possíveis efeitos de características dos conjuntos de formas e caracteres escolhidos (ao invés das condições experimentais).

Tabela 1. Valores de FMR, PRForma, PRPos e PRCor em cada uma das condições experimentais do Experimento 1

\begin{tabular}{ccccc}
\hline Condição & FMR & PRForma & PRPos & PRCor \\
\hline 1 & 1,00 & 0,13 & 1,00 & 0,13 \\
2 & 1,00 & 1,00 & 0,13 & 0,13 \\
3 & 1,00 & 0,25 & 0,50 & 0,13 \\
4 & 1,00 & 0,50 & 0,25 & 0,13 \\
5 & 1,00 & 0,25 & 0,25 & 0,25 \\
\hline
\end{tabular}

Com base nas análises da complexidade das tarefas (Oliveira-Castro \& cols., 1999; Oliveira-Castro \& cols., 2002) planejadas para cada uma das condições experimentais, a Tabela 1 mostra os valores de FMR (freqüência média programada de reforço), PRForma (probabilidade programada de reforço dada uma forma), PRPos (probabilidade programada de reforço dada uma posição) e PRCor (probabilidade programada de reforço dada uma cor). Portanto, como pode ser observado na Tabela 1, o valor de FMR foi igual a 1,00 para todas as condições experimentais, já que em todas elas havia um reforço programado para cada uma das oito respostas diferentes a serem aprendidas (oito caracteres) em cada bloco de tentativas, ou seja, nenhum dos oito caracteres a serem aprendidos se repetia durante um bloco de tentativas. De acordo com o delineamento, a comparação dos desempenhos obtidos nas Condições 1 e 2 possibilitava investigar os efeitos diferenciais de PRForma e PRpos, ou seja, tal comparação permitia verificar se o desempenho é melhor quando o participante deve aprender uma seqüência de oito respostas diferentes na presença de um único estímulo discriminativo (Condição 1) ou quando o participante deve aprender uma resposta na presença de cada um de oito estímulos discriminativos (Condição 2). A comparação do desempenho nas Condições 3 e 4 possibilitava testar esse mesmo efeito diferencial de PRForma e PRPos, sendo que nesse caso a comparação era entre aprender quatro respostas diferentes na presença de dois estímulos discriminativos (Condição 3) ou aprender duas respostas diferentes na presença de cada um de quatro estímulos discriminativos (Condição 4). Considerando que resultados anteriores indicaram maiores efeitos de mudanças em PRForma do que em PRPos (Oliveira-Castro \& cols., 2002), poder-se-ia esperar melhor desempenho na Condição 2 quando comparada com a 1 e melhor desempenho na Condição 4 quando comparada com a 3. Além disso, o delineamento possibilitou testar os possíveis efeitos de aumento no número de dimensões discriminativas, já que as Condições 4 e 5 eram ambas compostas por quatro pares com dois caracteres cada, sendo que as duas diferiam apenas quanto ao número de dimensões discriminativas na tarefas, a saber, duas (forma e posição na Condição 4) ou três (forma, posição e cor na Condição 5). O valor de PRCor foi obtido de forma análoga aos valores de PRForma e PRPos, ou seja, pela divisão da freqüência média de reforço dada uma cor pelo número de respostas corretas possíveis dada uma cor. No caso das quatro primeiras condições esse valor foi igual a 0,13 , já que, sendo todas as formas da mesma cor, a freqüência média programada de reforço na presença da cor era igual a 1 (i.e., cada resposta seria correta apenas uma vez em cada bloco de tentativas) e o número de respostas corretas possíveis dada uma cor era 8 (i.e., todas as oito respostas seriam corretas na presença da mesma cor). Portanto, PRCor foi igual a $1 / 8$, ou seja, 0,13 . Na Condição 5 , a freqüência média programada de reforço dada uma cor foi também igual a 1,enquanto o número de respostas corretas possíveis dada uma cor foi igual a 4 (PRCor foi obtido por 1/4).

\section{Resultados e Discussão}

Os parâmetros da Equação 1 foram calculados para cada uma das condições experimentais para cada um dos participantes e indicaram um valor médio de $r^{2}$ igual a $0,69(D P$ $=0,22, n=30$ ), sendo que mais da metade dos valores foi maior que 0,80 . Esses resultados sugerem um bom ajuste dos dados à Equação 1, muito semelhante aos relatados na literatura (Oliveira-Castro \& cols., 1999; Oliveira-Castro \& cols., 2002), o que justifica a utilização da equação para descrever o desempenho dos participantes.

A Figura 2 apresenta a área da função (i.e., tempo total estimado de precorrente auxiliar por corretas), calculada com base na Equação 1, para cada condição experimental e cada participante. $O$ valor de área foi menor na Condição 2 do que na Condição 1 para cinco dos seis participantes, e foi menor na Condição 4 do que na Condição 3 também para cinco participantes. Considerando que, em ambas as comparações, os melhores desempenhos entre as condições estiveram associados a maiores valores de PRForma, esses resultados
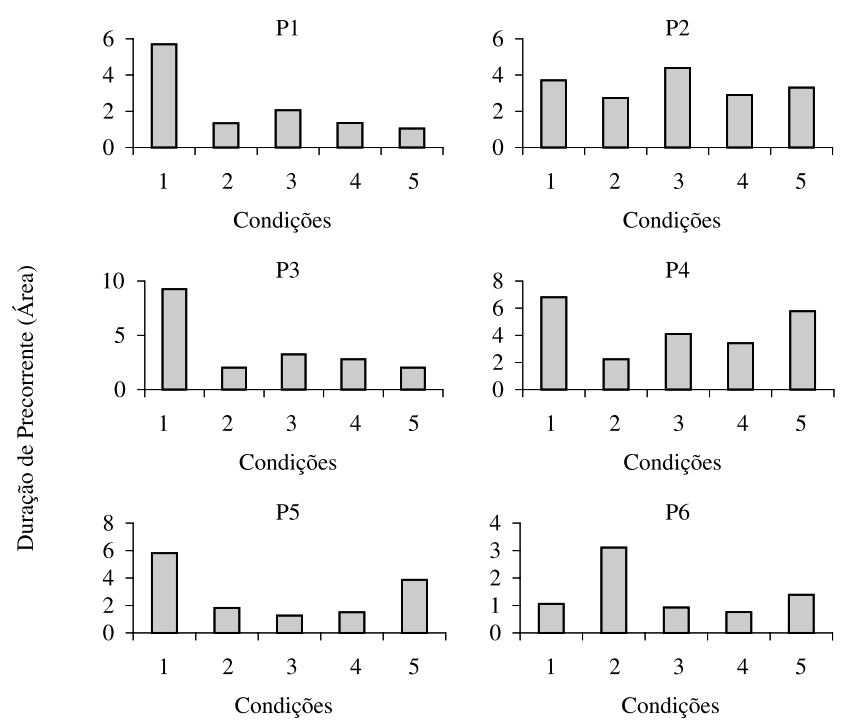

Figura 2. Valores de área (Equação 1) em cada condição experimental para cada participante do Experimento 1 
corroboram aqueles relatados na literatura (Oliveira-Castro $\&$ cols., 2002), os quais sugerem que mudanças nos valores de PRForma têm efeitos mais acentuados que mudanças nos valores de PRPos.

No presente caso, tais resultados sugerem ainda que o desempenho foi melhor (i.e., menos tempo de auxílio) na condição em que uma resposta diferente estava associada a cada uma de oito formas diferentes (Condição 2) do que na condição em que oito respostas diferentes estavam todas emparelhadas com uma única forma (Condição 1). Do mesmo modo, o desempenho foi melhor na condição em que duas respostas diferentes estavam emparelhadas a quatro formas (Condição 4) do que quando quatro respostas diferentes estavam emparelhadas a duas formas (Condição 3). Tomados em conjunto, esses resultados sugerem que o tempo total de precorrente auxiliar necessário para aprender todos os pares diminui com a diminuição do número de respostas emparelhadas com cada uma das formas, ou seja, quanto mais unívoco for o emparelhamento de formas e respostas, mas rápida será a diminuição da resposta precorrente. Para testar essa conclusão geral, uma correlação (Pearson) foi calculada entre o número de respostas emparelhadas com cada uma das formas (i.e., 8 na Condição 1, 4 na Condição 3, 2 na Condição 4, e 1 na Condição 2) e a área da função obtida para cada um dos participantes. Essa correlação foi significativa e igual a 0,63 $(\mathrm{n}=24, p=0,001)$, corroborando tal conclusão.

Como mencionado anteriormente, a comparação dos desempenhos nas Condições 4 e 5 possibilita examinar o efeito do número de dimensões discriminativas, na medida em que ambas eram compostas por quatro pares com duas respostas diferentes cada e diferiam apenas quanto ao número de dimensões (forma e posição versus forma, posição e cor). Como mostra a Figura 2, a área obtida na Condição 5 foi maior que aquela obtida na Condição 4 para quatro dos seis sujeitos, sugerindo que o aumento de dimensões discriminativas pode produzir um aumento nos valores de área. Tendo em vista o pequeno número de participantes nesse experimento, um segundo experimento foi conduzido com o objetivo de examinar mais detalhadamente esse possível efeito.

\section{Experimento 2}

Com o propósito de testar mais sistematicamente a possível influência do número de dimensões discriminativas sobre a diminuição do comportamento precorrente auxiliar, em diferentes condições do Experimento 2 o número de dimensões foi igual a dois (forma e posição: Condição 1), três (forma, posição e cor: Condições 2 e 3 ) ou quatro (forma, posição, cor, e forma secundária: Condição 4) dimensões.

\section{Método}

\section{Participantes}

Participaram do experimento 16 alunos da Universidade de Brasília (13 mulheres), com idade média de 19,06 anos $(D P=1,57)$, que receberam, para cada hora de participação, 0,5\% da menção final em disciplinas do Departamento de Processos Psicológicos Básicos do Instituto de Psicologia (até o máximo de 5\%).

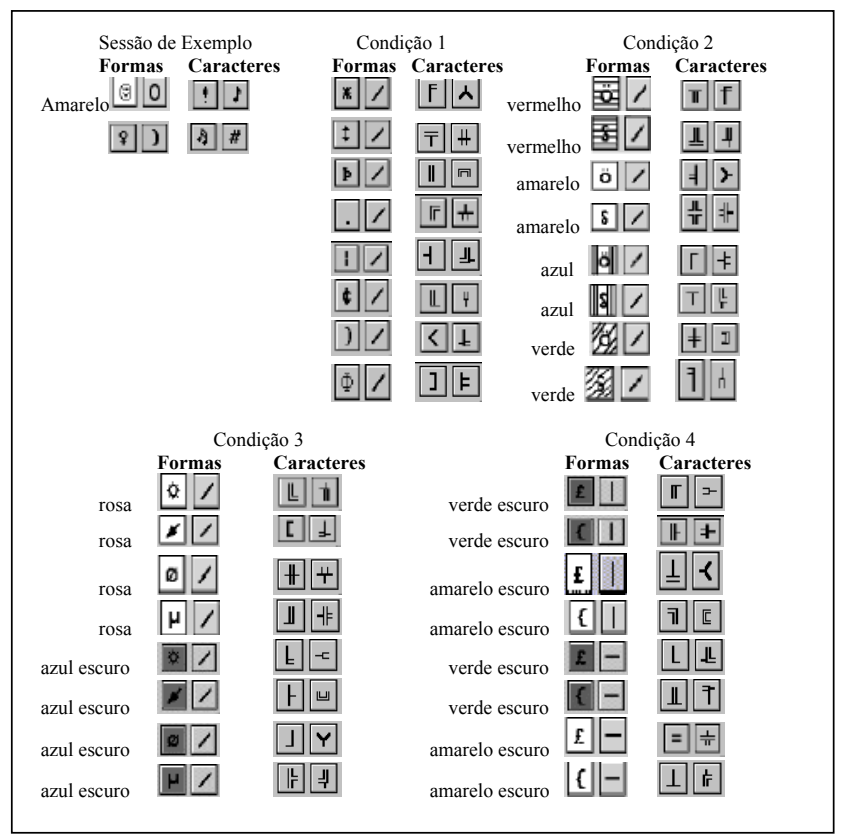

Figura 3. Pares de formas e caracteres utilizados no Experimento 2. Os nomes de cores ao lado das formas indicam a cor do fundo da respectiva forma.

\section{Material}

O material foi muito parecido com aquele adotado no Experimento 1, sendo que a Figura 3 apresenta as formas e conjuntos de caracteres usados para compor os pares associados em cada uma das condições experimentais. Os primeiros membros dos pares foram formados por duas formas, sendo que aquelas que apareciam do lado direito serão denominadas de formas secundárias, para facilitar a exposição.

\section{Procedimento}

De forma geral, o procedimento foi idêntico àquele adotado no Experimento 1, com as seguintes modificações: 1) os 16 participantes foram divididos em oito grupos de dois participantes; 2) quatro condições experimentais foram conduzidas, cada uma em uma sessão, as quais foram apresentadas em seqüências e dias diferentes para cada grupo experimental; 3) adotou-se um balanceamento incompleto para a ordem de apresentação das sessões para os grupos, sendo que cada condição foi apresentada duas vezes em cada uma das quatro posições na sequiência de sessões; 4 ) as condições experimentais foram diferentes e estão descritas a seguir.

A Figura 3 apresenta os pares de formas e caracteres utilizados em cada uma das condições experimentais. Todas as condições experimentais foram constituídas por tarefas com oito pares associados. Os primeiros membros de todos os pares em todas as condições foram formados por dois caracteres, denominados aqui de "forma" (à esquerda) e "forma secundária" (à direita). Os segundos membros de todos os pares de todas as condições foram formados por dois caracteres arbitrários, o que justifica a afirmação feita anteriormente de que uma das dimensões discriminativas das tarefas era a posição dos caracteres no conjunto a ser digitado, apesar dessa dimensão ter permanecido igual para todas 
as condições. As quatro condições experimentais diferiam, portanto, apenas com base nas dimensões discriminativas relacionadas aos primeiros membros dos pares.

Como mostra a figura, os primeiros membros dos pares da Condição 1 diferiam entre si com base apenas na forma de cada par, sendo que todas tinham a mesma cor e eram acompanhadas da mesma forma secundária (i.e., quadrado contendo uma linha diagonal inclinada para a direita). Na Condição 2, os primeiros membros dos oito pares diferiam entre si com relação à forma e à cor da forma, sendo cada um formado por uma das combinações de duas formas e quatro cores (as formas secundárias eram todas iguais). $\mathrm{Na}$ Condição 3 , os primeiros membros dos oito pares diferiam entre si com respeito à forma e à cor da forma, sendo cada um formado por uma das combinações de quatro formas e duas cores (novamente as formas secundárias eram todas iguais). Na Condição 4 , os primeiros membros dos oito pares diferiam entre si com relação à forma, cor da forma e forma secundária. Cada um dos primeiros membros foi formado por uma de duas formas (i.e., "parágrafo" ou "chave esquerda"), uma de duas cores de forma (i.e., verde escuro ou amarelo escuro), e uma de duas formas secundárias (i.e., "linha vertical" ou "linha horizontal").

A Tabela 2 mostra os valores de FMR, PRForma, PRFormaSec (i.e., forma secundária), PRPos e PRCor para cada uma das condições do Experimento 2. Como pode ser observado, as condições diferem entre si com relação ao número de dimensões discriminativas dos primeiros membros dos pares, ou seja, Condição 1 com apenas uma dimensão (formas), Condições 2 e 3 com duas dimensões (formas e cores), e Condição 4 com três dimensões (formas, cores, e formas secundárias). Assumindo que as probabilidades programadas de reforço para diferentes dimensões discriminativas podem ser calculadas linearmente (i.e., todas tendo o mesmo peso), a média calculada com a soma de PRForma, PRFormaSec e PRCor para cada uma das condições são também apresentadas na Tabela 2. Se essa premissa estiver correta, tendo em vista os valores das médias obtidas, poder-se-ia esperar um melhor desempenho na Condição 1, seguido de desempenho semelhante nas Condições 2 e 3, e finalmente um pior desempenho na Condição 4.

\section{Resultados e Discussão}

Os parâmetros da Equação 1 foram calculados para cada condição experimental de cada participante e indicaram um valor médio de $r^{2}$ igual a $0,75(D P=0,17, n=64)$. Esse valor é um pouco maior do que aqueles relatados em experimentos anteriores (Oliveira-Castro \& cols., 1999; Oliveira-Castro \& cols., 2002) e sugere um bom ajuste dos dados à equação, justificando o uso da mesma como medida de desempenho.

Os valores de área (Equação 1) obtidos para cada participante nas Condições $2(M=2,10, D P=0,87, n=16)$ e $3(M=2,20, D P=0,97, n=16)$ foram muito semelhantes, sugerindo que não houve diferenças de desempenho nas duas condições. Um teste $t$, comparando a área das duas condições, não mostrou diferenças significativas entre as médias das áreas $(t=0,34, d f=15, p=0,74)$. Tendo em vista que os primeiros membros dos pares em ambas as condições diferiam com respeito a duas dimensões (i.e., forma e cor) e que não houve diferença de desempenho entre elas, as duas condições passaram a ser tratadas como uma única condição (i.e., Condição 23). Para isso, a média dos valores de área obtidos nas duas condição foi calculada $(M=2,15, D P=$ $0,74)$ para que fosse comparada com as áreas obtidas nas outras duas condições.

Tabela 2. Valores de FMR, PRForma, PRFormaSec, PRPos, PRCor e média de PRPrimeiroMembro (i.e., (PRForma + PRFormaSec + PRCor) / 3), em cada uma das condições experimentais do Experimento 2

\begin{tabular}{ccccccc}
\hline Condição & FMR & PRForma & PRFormaSec & PRPos & PRCor & Média \\
\hline 1 & 1,00 & 0,50 & 0,06 & 0,13 & 0,06 & 0,21 \\
2 & 1,00 & 0,13 & 0,06 & 0,13 & 0,25 & 0,15 \\
3 & 1,00 & 0,25 & 0,06 & 0,13 & 0,13 & 0,15 \\
4 & 1,00 & 0,13 & 0,13 & 0,13 & 0,13 & 0,13 \\
\hline
\end{tabular}

A Figura 4 apresenta os valores de área em função da probabilidade média programada de reforço para as dimensões dos primeiros membros dos pares i.e., (PRForma + PRFormaSec + PRCor) / 3, ver Tabela 2), calculada para cada um dos participantes. Pode se observar na figura que, para 12 dos 16 participantes, o valor de área diminuiu com o aumento da probabilidade média de 0,13 (Condição 4) para 0,15 (Condição 23). Também para 12 participantes o valor da área diminuiu com o aumento da probabilidade média de 0,15 (Condição 23) para 0,21 (Condição 1). Um teste $t$ para amostras emparelhadas indicou que as diferenças entre os valores de área da Condição $4(\mathrm{M}=2,78, D P=1,69)$ e Condição 23 (t $(15)=1,958, p=0,069)$, da Condição $1(\mathrm{M}=$ $1,81, D P=0,65)$ e Condição $23(\mathrm{t}(15)=1,963, p=0,068)$, e da Condição 4 e Condição $1(\mathrm{t}(15)=2,264, p=0,039)$ foram todas significativas $(p<0,10)$. Uma análise de correlação (Pearson) entre os valores de área e a probabilidade média se mostrou significativa $(r=-0,264, \mathrm{n}=64, p=0,035)$, confirmando a tendência de diminuição nos valores de área com o aumento da probabilidade média programada de reforço para as dimensões dos primeiros membros dos pares.
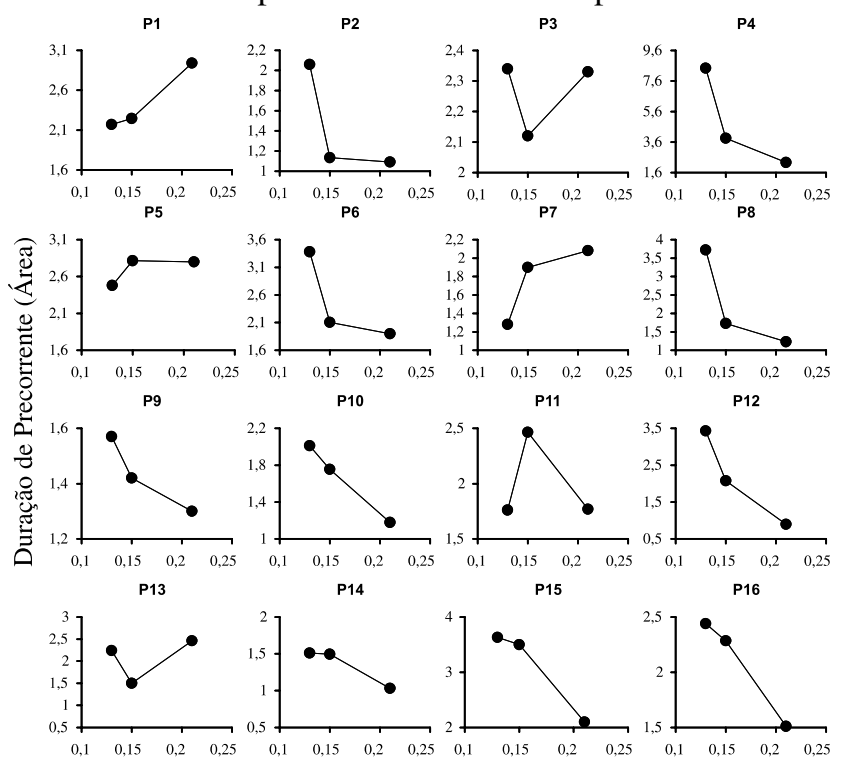

(PRForma + PRFormaSec + PRCor) $/ 3$

Figura 4. Valores de área (Equação 1) como função da probabilidade média programada de reforço para as dimensões dos primeiros membros dos pares (i.e., (PRForma + PRFormaSec + PRCor) /3, ver Tabela 2) em cada condição experimental para cada participante do Experimento 2 


\section{Considerações Finais}

Os resultados do Experimento 1 sugerem que a aprendizagem de um número $n$ de respostas se dá mais rapidamente quando cada resposta está associada a um evento ambiental específico do que quando elas têm que ser aprendidas em uma seqüência determinada (i.e., estando todas elas associadas ao mesmo evento ambiental). Dito de outra forma, diminuições nos valores de PRForma têm um efeito mais deletério sobre o desempenho do que iguais diminuições nos valores de PRPos, conclusão esta que corrobora resultados relatados na literatura, obtidos com diferentes tipos de manipulação dos valores de PRForma e PRPos (Oliveira-Castro \& cols., 1999; Oliveira-Castro \& cols., 2002).

Esse efeito diferencial de PRForma e PRPos pode se dever à forma como as consequiências para as respostas foram programadas na tarefa. No procedimento adotado, a consequiência indicando se a resposta estava correta ou incorreta (som agudo/grave e palavra "certo/errado" no monitor) ocorria após a "digitação" (com o mouse) da última resposta do conjunto de caracteres que compunham o segundo membro do par. No caso da tarefa com oito pares de forma-caractere, por exemplo, na qual, para cada forma havia um caractere que seria correto (i.e., Condição 2, Experimento 1), o feedback de "certo/errado" ocorria após a digitação de cada resposta. Já no caso da tarefa em que um único par de forma-caractere foi empregado, na qual uma única forma estava associada a uma sequiência de oito caracteres (i.e., Condição 1, Experimento 1), o feedback "certo/errado" ocorria somente após a digitação do oitavo caractere da sequiência. Essa menor densidade de feedback pode ser uma das variáveis que favoreceu o desempenho nas Condições 2 e 4 quando comparados com o desempenho nas Condições 1 e 3, respectivamente. Esse possível efeito de densidade de feedback poderia ser testado com um procedimento no qual o feedback "certo/errado" fosse fornecido após cada uma das respostas das seqüências de respostas nas condições com mais de um caractere por forma.

Caso esse efeito diferencial não deixe de ocorrer com a inclusão do feedback para cada resposta, uma outra possível explicação para os efeitos observados seria baseada nas diferentes relações entre eventos do ambiente, que passam a funcionar como eventos discriminativos, e cada resposta digitada. Nas tarefas nas quais apenas um caractere deve ser digitado frente a cada forma, cada resposta a ser aprendida na tarefa ficaria associada a um evento ambiental específico, isto é, a uma forma específica. Nas tarefas nas quais mais de um caractere deve ser digitado frente a cada forma, a forma passaria a funcionar como estímulo discriminativo para o primeiro membro da sequiência e, possivelmente, também para as outras respostas da sequiência. De acordo com a interpretação difundida de encadeamento (Skinner, 1953), cada resposta da sequiência poderia também gerar estímulos discriminativos para as respostas seguintes, na medida em que os caracteres digitados permaneciam no monitor até a digitação do número total de caracteres que compunham a seqüência. Esse tipo de explicação estaria baseada em possíveis diferenças na eficiência de associações baseadas em eventos produzidos independentemente do comportamento de quem desempenha a tarefa e de associações baseadas em eventos produzidos pelo comportamento da pessoa que desempenha a tarefa (i.e., deixas produzidas por respostas ou estímulos produzidos pelo próprio sujeito). Apesar da elucidação dos efeitos dessas variáveis dever aguardar futuras investigações planejadas explicitamente para separá-los, algumas recomendações práticas podem ser sugeridas com base nos resultados aqui obtidos. Uma das principais recomendações para técnicas de ensino seria programar, tanto quanto possível, relações unívocas (i.e., correspondência um-a-um) entre os eventos que devem funcionar como estímulos discriminativos e as respostas que devem ser aprendidas, e entre as respostas que devem ser aprendidas e as consequiências (i.e., "certo/errado") para as mesmas.

Os resultados dos dois experimentos também permitem concluir que o aumento no número de dimensões discriminativas dos primeiros membros dos pares, mantendo outras variáveis constantes, piorou o desempenho dos participantes, ou seja, produziu um aumento na duração de comportamento precorrente necessário para aprender a tarefa. Tais resultados sugerem que a aprendizagem de quatro respostas na presença de quatro eventos que diferem em termos de uma dimensão (por exemplo, quatro formas ou quatro cores) será mais rápida do que a aprendizagem de quatro respostas na presença de quatro eventos que diferem em termos de combinações de duas dimensões (por exemplo, duas formas em duas cores diferentes). Usando novamente o exemplo do número de telefone, os resultados sugerem que a aprendizagem de quatro números diferentes associados a quatro nomes diferentes seria mais rápida do que a aprendizagem de quatro números diferentes associados a dois nomes diferentes combinados com dois horários diferentes (por exemplo, dia e noite), tudo mais mantido constante.

Além disso, os efeitos decorrentes de mudanças no número de dimensões discriminativas da tarefa foram compatíveis com a quantificação das contingências programadas, sugerindo, dessa forma, uma explicação para o fenômeno. A diminuição no desempenho, associada a aumentos no número de dimensões discriminativas, seria explicada com base na diminuição das probabilidades programadas de reforço para as diferentes dimensões (i.e., PRForma, PRFormaSec, ou PRCor). Esses resultados podem ser interpretados como estendendo os achados relatados na literatura sobre controle discriminativo, particularmente aquela que trata de fenômenos tais como bloqueio (respondente, e.g., Rescorla \& Wagner, 1972; e operante, e.g., Vom Saal \& Jenkins, 1970). Em tais casos, quando uma mesma característica de um evento passa a ser emparelhada com mais de uma resposta, o valor preditivo do evento diminui ou, dito de outro modo, a probabilidade programada de reforço diminui. Esse tipo de análise pode ser aplicada a situações de ensino de leitura e escrita, principalmente àqueles casos nos quais uma mesma letra está associada a diferentes fonemas ou um fonema está associado a diferentes letras (Oliveira-Castro, 2000a).

\section{Referências}

Anderson, J. R. (1993). Rules of the mind. Hillsdale, NJ: Erlbaum.

Blessing, S. B. \& Anderson, J. R. (1996). How people learn to skip steps. Journal of Experimental Psychology: Learning, Memory and Cognition, 22(3), 576-598. 
Carvalho, G. P. (2000). Comportamento precorrente auxiliar em tarefas de recombinação de unidades menores: Efeitos da modalidade sensorial do estímulo. Dissertação de Mestrado, Universidade de Brasília, Brasília.

Coelho, D. S. (1996). Memorização: Efeitos da complexidade discriminativa da tarefa sobre a diminuição da resposta intermediária. Dissertação de Mestrado, Universidade de Brasília, Brasília.

Coelho, D. S. (2000). Comportamento precorrente auxiliar: Efeitos de contingências programadas de reforço no treino sobre o desempenho em sessões de recombinação. Tese de Doutorado, Universidade de Brasília, Brasília.

Ferreira, D. C. S. (2001). Efeitos de indução de erros sobre o comportamento precorrente auxiliar em uma tarefa de recombinação de unidades menores. Dissertação de mestrado, Universidade de Brasília, Brasília.

Flores, E. P. (1997). Comportamento auxiliar em tarefas sucessivas: Efeitos de mudanças no estímulo e/ou na resposta exigida. Dissertação de Mestrado, Universidade de Brasília, Brasília.

Flores, E. P. (2003). "Saber como" e "saber sobre" em uma tarefa de pares associados: Efeitos da complexidade da tarefa e das instruções. Tese de Doutorado, Universidade de Brasília, Brasília.

Gosch, C. S. (2000). Comportamento precorrente auxiliar: Efeitos da quantidade de treino sobre a simulação de leitura generalizada. Dissertação de Mestrado, Universidade de Brasília, Brasília.

Oliveira-Castro, J. M. (1992). "Fazer na cabeça": uso metafórico e negativo. Psicologia: Teoria e Pesquisa, 8(2), 267-272.

Oliveira-Castro, J. M. (1993). "Fazer na cabeça": Análise conceitual, demonstrações empíricas e considerações teóricas. Psicologia USP, 4 (1/2) :171-202.

Oliveira-Castro, J. M. (2000a). Contingências programadas de reforço e complexidade discriminativa de tarefa: Aplicações a situações de ensino de leitura. Em R. C. Wielenska (Org.), Sobre comportamento e cognição (v. 6): Questionando e ampliando a teoria e as intervenções clínicas e em outros contextos (pp. 111-120). Santo André, SP: SET.

Oliveira-Castro, J. M. (2000b). The negative function of 'doing in the head' and behavioristic interpretations of private events. Mexican Journal of Behavior Analysis, 26(1), 1-25.

Oliveira-Castro, J. M., Carvalho, G. P., Faria, J. B., Dias, M. B., Melo, C. S. C. A. \& Alves, C. B. (1998a). Efeitos da freqüência programada de reforço para seqüências de respostas sobre a diminuição da resposta precorrente auxiliar em uma tarefa de pares associados. Trabalho apresentado na XXVIII Reunião Anual da Sociedade Brasileira de Psicologia, Ribeirão Preto, SP.

Oliveira-Castro, J. M., Carvalho, G. P., Faria, J. B., Dias, M. B., Melo, C. S. C. A. \& Alves, C. B. (1998b). Efeitos do número de posições sobre a diminuição da resposta precorrente auxiliar em uma tarefa de pares associados. Trabalho apresentado na XXVIII Reunião Anual da Sociedade Brasileira de Psicologia, Ribeirão Preto, SP.
Oliveira-Castro, J. M., Castilho, G. M., Faria, J. B., Brasileiro, F. C., Dias, M. B. \& Hostensky, E. L. (1997). Efeitos do número de pares sobre a diminuição da resposta auxiliar. Trabalho apresentado na XXVII Reunião Anual da Sociedade Brasileira de Psicologia, Ribeirão Preto, SP.

Oliveira-Castro, J. M., Coelho, D. S., Faria, J. B., Oliveira, G. S., Schwarz, E., Medeiros, C. A., Lemes, W. R., Brasileiro, F. C. \& Morais, F. P. (1996a). Efeitos do número de respostas na tarefa sobre a diminuição da resposta auxiliar. Trabalho apresentado na XXVI Reunião Anual da Sociedade Brasileira de Psicologia, Ribeirão Preto, SP.

Oliveira-Castro, J. M., Coelho, D. S., Faria, J. B., Oliveira, G. S., Schwarz, E., Medeiros, C. A., Lemes, W. R., Brasileiro, F. C. \& Morais, F. P. (1996b). Efeitos da correlação resposta/posição sobre a diminuição da resposta auxiliar. Trabalho apresentado na XXVI Reunião Anual da Sociedade Brasileira de Psicologia, Ribeirão Preto, SP.

Oliveira-Castro, J. M., Coelho, D. S. \& Oliveira-Castro, G. A. (1999). Decrease of precurrent behavior as training increases: Effects of task complexity. The Psychological Record, 49, 299-325.

Oliveira-Castro, J. M., Faria, J. B., Dias, M. B. \& Coelho, D. S. (2002). Effects of task complexity on learning to skip steps: An operant analysis. Behavioural Processes, 59, 101-120.

Rescorla, R. A. \& Wagner, A. R. (1972). A theory of Pavlovian conditioning: Variations in the effectiveness of reinforcement and nonreinforcement. Em A. H. Black \& W. F. Prokasy (Orgs.), Classical conditioning II (pp. 64-99). New York: AppletonCentury-Crofts.

Skinner, B. F. (1953). Science and human behavior. New York: The Macmillan Company.

Skinner, B. F. (1957). Verbal behavior. Englewood Cliffs, NJ: Prentice-Hall, Inc.

Skinner, B. F. (1968). The technology of teaching. Englewood Cliffs, NJ: Prentice-Hall.

Skinner, B. F. (1969). Contingencies of reinforcement: A theoretical analysis. Englewood Cliffs, NJ: Prentice-Hall, Inc.

Souza, C. B. A. (1997). Comportamento auxiliar: Efeitos do parcelamento de uma tarefa com diferentes complexidades discriminativas. Dissertação de Mestrado, Universidade de Brasília, Brasília.

Vom Saal, W. \& Jenkins, H. M. (1970). Blocking the development of stimulus control. Learning and Motivation, 1(1), 52-64. 